
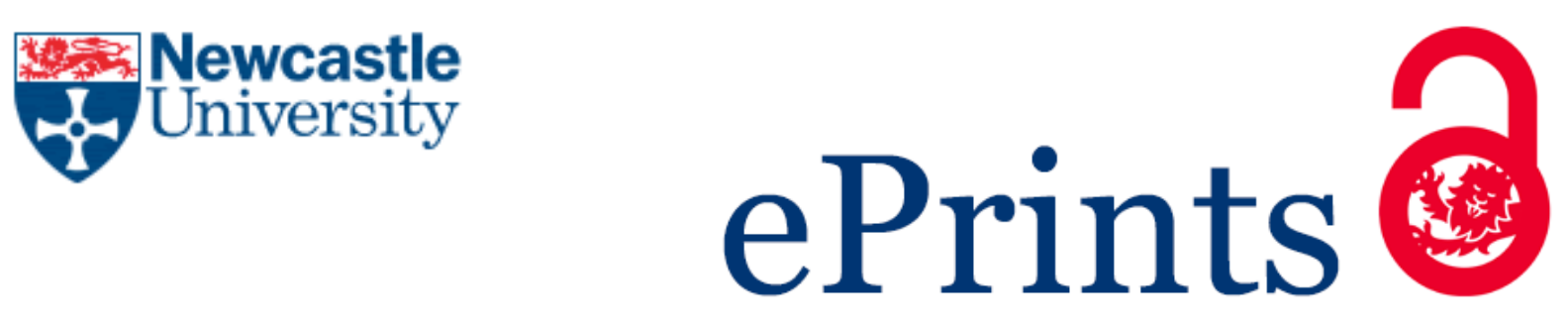

Wong A, Seger DL, Slight SP, Amato MG, Beeler PE, Fiskio JM, Bates DW. Evaluation of 'Definite' Anaphylaxis Drug Allergy Alert Overrides in Inpatient and Outpatient Settings.

Drug Safety 2018, 41(3), 297-302.

\title{
Copyright:
}

The final publication is available at Springer via https://doi.org/10.1007/s40264-017-0615-1

Date deposited:

$06 / 10 / 2017$

Embargo release date:

09 November 2018

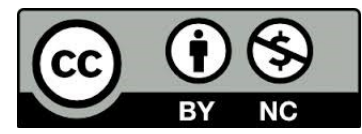

This work is licensed under a Creative Commons Attribution-NonCommercial 3.0 Unported License 
Draft June 9, 2017

\section{Evaluation of 'Definite' Anaphylaxis Drug Allergy Alert Overrides in Inpatient and Outpatient Settings}

Adrian Wong, PharmD, MPH ${ }^{1,2}$

Diane L. Seger, $\mathrm{RPh}^{1,3}$

Sarah P. Slight, MPharm, PhD, PGDip ${ }^{1,4,5}$

Mary G. Amato, PharmD, MPH ${ }^{1,2}$

Patrick E. Beeler, MD ${ }^{1,6,7}$

Julie M. Fiskio, BS ${ }^{1,3}$

David W. Bates, MD, MSc ${ }^{1,7}$

${ }^{1}$ The Center for Patient Safety Research and Practice, Division of General Internal Medicine and Primary Care, Brigham and Women's Hospital, Boston, MA, USA

${ }^{2}$ MCPHS University, Boston, MA, USA

${ }^{3}$ Clinical \& Quality Analysis, Partners HealthCare, Somerville, MA, USA

${ }^{4}$ School of Medicine, Pharmacy and Health, The University of Durham, Stockton on Tees, Durham, UK

${ }^{5}$ Newcastle upon Tyne Hospitals NHS Foundation Trust, Newcastle, United Kingdom

${ }^{6}$ Research Center for Medical Informatics, University Hospital, Zurich, Switzerland

${ }^{7}$ Harvard Medical School, Boston, MA, USA

Corresponding author:

David Bates, MD, MSc

Chief, Division of General Internal Medicine and Primary Care

Brigham and Women's Hospital

1620 Tremont Street

75 Francis Street

Boston, MA 02115

Phone: 617-732-5650 
Fax: 617-732-7072

Email: dbates@bwh.harvard.edu

\section{Disclosures}

Dr. Bates reported receiving equity from Intensix, which makes software to support clinical decision-making in intensive care; being named as coinventor on patent No. 6029138 held by Brigham and Women's Hospital on the use of decision support software for medical management, licensed to the Medicalis Corporation, and holding a minority equity position in Medicalis, which develops web-based decision support for radiology test ordering; consulting for EarlySense, which makes patient safety monitoring systems; receiving equity and cash compensation from QPID Inc, a company focused on intelligence systems for electronic health records; receiving cash compensation from CDI (Negev) Ltd, which is a not-for-profit incubator for health IT startups; receiving equity from Enelgy, which makes software to support evidencebased clinical decisions, from Ethosmart, which makes software to help patients with chronic diseases, and from MDClone, which takes clinical data and produces deidentified versions of it. The remaining authors have disclosed that they do not have any conflicts of interest.

This study was funded by grant \#U19HS021094 from the Agency for Healthcare Research and Quality (AHRQ). Dr. Beeler was supported by the Swiss National Science Foundation.

Abstract (272), Body (2312) 


\section{ABSTRACT}

Background: Drug allergy interaction (DAI) alerts are generated when a known adverse sensitivity-inducing substance is prescribed. A recent study at our institution showed that providers overrode most DAI alerts, including those that warned against a potentially lifethreatening 'anaphylaxis.'

Objective: To determine the rate of anaphylaxis overrides, the reasons for these overrides, whether the overrides were appropriate, and if harm occurred from overrides.

Methods: All DAI alerts, with a reaction of 'anaphylaxis', were analyzed for inpatients and outpatients within our health-system between January 2009 and December 2011. Only alerts that were triggered by 'definite' matches (i.e., same ordered medication as documented allergen) were included. A total of 202 inpatient and 16 outpatient alerts met inclusion criteria, and respective charts were reviewed to assess the appropriateness of overrides and potential harm induced by the drug.

Results: The rate of overrides for 'definite' anaphylaxis DAI alerts was high (inpatient: $\mathrm{n}=93$, $46.0 \%$; outpatient: $n=11,68.8 \%)$ but appropriate for most overrides in the inpatient $(n=78$, 83.9\%) and outpatient setting $(n=11,100 \%)$. Desensitizations accounted for the majority of appropriate overrides $(n=65,73.0 \%)$. The most common override reasons in the inpatient and outpatient setting were 'administer per desensitization protocol' $(\mathrm{n}=64,82.1 \%)$ and 'patient does not have this allergy' $(n=7,63.6 \%)$, respectively, and all of the latter were removed from the patients' records. No harm was associated with appropriate or inappropriate overrides in either setting, particularly because many drugs were not administered eventually. 
Conclusions: Overrides of 'definite' anaphylaxis DAI alerts were common but a large fraction was appropriate. Most overrides were due to desensitizations and removal of these alerts from order sets would substantially reduce the alert burden. Allergy reconciliation for patients could further improve the specificity of potentially critical decision support. 


\section{BACKGROUND}

Adverse drug reactions (ADRs) are responses to medications at normal human doses, which are noxious and unintended. ${ }^{1}$ Drug allergies represent one type of ADRs; clinically both sensitivities and allergies are often reported as allergies by clinicians. However, true allergies are immunologically-mediated hypersensitivity reactions that can range from mild reactions (e.g. itching) to severe reactions, such as anaphylaxis. ${ }^{2}$ These reactions account for less than $10 \%$ of ADRs. ${ }^{3}$ A large epidemiologic study of patient-reported allergy within our health-system found that $35.5 \%$ of our patients had documentation of at least one allergy, with $54.7 \%$ of this population only having one allergy documented. ${ }^{4}$ Anaphylaxis, which is a sudden onset of a lifethreatening systemic or hypersensitivity reaction, has been estimated to have a lifetime risk of $1.6 \%$ in the general population. ${ }^{5}$ The true incidence of medication-induced anaphylaxis is unknown, with variability based on medications, patient risk factors, and populations. ${ }^{3}$ Antibiotics such as penicillin represent a common documented allergen but most lack of objective evidence of reactions (i.e. immunologic testing). ${ }^{5}$ To deliver appropriate decision support in electronic health records (EHRs), it is important to accurately determine what the reaction was, to assess whether or not it appeared to be a true allergy, and to assess whether or not anaphylaxis was present. ${ }^{6}$

Nearly all EHRs include 'allergy' modules for documentation of a patient's reported and observed reactions to medications, and this is required for EHRs to be certified in the U.S. ${ }^{7}$ However, not all of these EHRs separate anaphylaxis from other types of reactions. A recent study conducted at our institution showed that the majority of reported reactions was commonly entered as "unknown." 
Given the severity of anaphylaxis, it would be expected that such CDS alerts would rarely be overridden; however, data suggest otherwise, with data identifying an override rate of approximately $80 \% .^{9}$ Some of the reasons for this include poor allergy record stewardship and alert fatigue ${ }^{8-10}$ In this study, we assessed 1) how many alerts related to anaphylaxis were present and how often they were overridden, 2) the reasons that providers overrode them, 3) the appropriateness of these overrides, and 4) harm that occurred upon administration of an overridden alert.

\section{METHODS}

Drug-allergy interaction (DAI) alerts with a documented reaction of 'anaphylaxis' in a patient's medical record were included from January 2009 to December 2011 for inpatients at Brigham and Women's Hospital (BWH) and for outpatients from primary care practices affiliated with Partners HealthCare. Partners HealthCare is an integrated healthcare delivery system in the greater Boston area that was founded by BWH and Massachusetts General Hospital (MGH), both large academic tertiary care centers. A patient's allergy information is entered in the EHR, which is then stored in a health-system wide repository, allowing for longitudinal record access. ${ }^{11}$ Only drug allergies identified as a 'definite' match between the ordered medication and the documented allergen were included in this analysis. These alerts were chosen given the highly sensitive mapping of medication cross-reactivity which has been identified in the literature. ${ }^{8}$

When a medication is ordered, it is compared to a patient's reported allergies using a commercial knowledge base (First DataBank, South San Francisco, CA). If there is a potential interaction between this documentation and the ordered medication, a DAI alert fires and is displayed on the provider's computer screen. These alerts are interruptive, requiring a provider to 
either cancel the order or to override the alert. In the inpatient setting, providers can see the documented reaction, reason for a previous override of this alert for a patient, and input a freetext reason for overriding this alert (Figure 1). Pharmacists and nurses can then see the DAI alert for those that were overridden, to allow for double-checking the order upon verification and administration. In the outpatient setting, providers can see the documented reaction and choose to keep the order through one of the following mechanisms, 1. Choose "Patient does not have this allergy, will D/C pre-existing allergy" or 2. Choose a coded reason for override (i.e., no reasonable alternatives) or 3. Input a free-text reason for the override (Figure 2).

Data included the ordered medication and the documented allergen in the patient's medical record that triggered the DAI alert, and the documented reason for override. We also collected serum tryptase levels to help with confirmation of actual anaphylaxis to the documented allergen near the time of allergy documentation, if available. ${ }^{5}$ The appropriateness of the override was assessed through detailed chart review according to a priori criteria developed by a multidisciplinary group. The criteria covered the evaluation of the documented override reason, evidence of previous tolerance of the medication, and if the medication was ordered for a desensitization by an allergy specialist. In evaluating the override coded reason 'Physician aware,' we evaluated documented notes in the patient record demonstrating that the medical team was actually aware that the patient had a medication ordered, despite a documented reaction of 'anaphylaxis', or if the patient had previously tolerated the medication.

\section{RESULTS}

A total of 1,851 inpatient and 216 outpatient DAI alerts with a reaction of 'anaphylaxis,' were included. These overrides were obtained from 499 inpatient encounters with a median number of 1 alert per encounter (IQR 1, 4), and 140 outpatient visits with a median number of 1 
alert per visit (IQR 1,2). The override rates for all alerts were $68.7 \%$ and $59.3 \%$ in the inpatient and outpatient settings, respectively. Demographics of patients are detailed in Table 1.

After reviewing alert content for non-'definite' matches, a total of $218(10.5 \%)$ alerts remained (inpatient, $\mathrm{n}=202,10.9 \%$; outpatient, $\mathrm{n}=16,7.1 \%$ ). The largest category of exclusion was that of antimicrobials and cross-sensitivity between the allergen and the ordered medication $(\mathrm{n}=795,43.0 \%)$. The group of 'definite' overrides were derived from 107 inpatient encounters with a median number of 1 alert per encounter (IQR 1,2) and 13 outpatient visits with a median number of 1 alert per visit (IQR 1, 1). The rate of 'definite' anaphylaxis DAI alert overrides were $46.0 \%(\mathrm{n}=93)$ and $68.8 \%(\mathrm{n}=11)$ in the inpatient and outpatient settings, respectively. The most common allergens associated with medication actions or overrides are detailed in Table 2 and Table 3 for the inpatient and outpatient settings, respectively. We were unable to obtain serum tryptase levels for any patient at the time of allergy documentation to help with confirmation of anaphylaxis to the documented allergen. This was due to no serum tryptase being ordered on the day of allergy documentation.

In evaluating the 93 overrides in the inpatient setting, aspirin was the most common allergen ( $\mathrm{n}=13,14.0 \%)$, with chemotherapy agents and antimicrobials the most common medication classes of allergens overridden ( $\mathrm{n}=49,52.7 \%$ and $\mathrm{n}=9,9.7 \%$, respectively). A total of 78 of the 93 overrides were identified to have been appropriate overrides (83.9\%). Of the 78 appropriately overridden alerts, 67 resulted in medication administration (85.9\%), with a median number of 1 administration (IQR 1,1 ). Only 2 of the appropriately overridden alerts resulted in eventual discontinuation of the allergen (2.6\%), which was not accomplished during the patient's stay. The override reasons for these cases were "Pt allergic to IV contrast not Readicat" and "Physician aware." Of the appropriate overrides that resulted in medication administration, 57 
had a documented override reason of 'desensitization' (85.1\%). Only 1 of these was not ordered via a desensitization protocol (1.8\%). No patient harm was found to have occurred following medication administration of the non-desensitization orders. Of the 15 inappropriately overridden DAI alerts in the inpatient setting, "Physician aware" was the most common reason for the override ( $\mathrm{n}=6,40.0 \%$ ), with "Low risk cross sensitivity" being the second most common $(n=3,20.0 \%)$. Twelve of the inappropriately overridden alerts did not pass order verification by the pharmacist either due to pharmacist intervention or from the provider subsequently discontinuing the order after order entry. The remaining 3 overrides were for prochlorperazine suppositories ordered as needed, which the patient was never administered.

In evaluating the 11 overrides in the outpatient setting, all were identified to be appropriately overridden (100\%). Aspirin was the most commonly overridden allergen $(n=5$, $55.5 \%)$, followed by morphine $(\mathrm{n}=2,22.2 \%)$. The most common reason for an appropriate override was "Patient does not have this allergy - will D/C pre-existing allergy." In all of these cases, the allergen was removed from the patient's medical record.

\section{DISCUSSION}

We assessed how often anaphylaxis was presented as the potential reaction when allergy warnings were displayed, how often warnings were overridden, and what the clinical consequences of these overrides were for the patient. We found that while overrides were common, over $80 \%$ of overrides were appropriate, and we found no overrides that induced harm including inappropriately overridden alerts as no medication administration occurred from these alerts. Many of these warnings occurred in the context of desensitization protocols that usually require additional knowledge and attention by specialists. Overall, these data suggest that while 
anaphylaxis can be very serious, clinicians in this institution appeared appropriately concerned about it and were making good clinical decisions.

Overrides of DAI alerts have been identified as common in previous studies, with rates ranging from $80 \%$ to $96 \%{ }^{8-11}$ However, according to our data, anaphylaxis seems to be a rare type of allergic reaction. Override rates for anaphylaxis alerts for any DAI (i.e. not specific to 'definite' matches) were previously found to be $81.9 \%$ and $77.0 \%$ in the inpatient and outpatient settings, respectively. ${ }^{8}$ These findings differ from ours, which were higher in the outpatient setting. On one hand, it might be expected that override rates of 'anaphylaxis' alerts would be lower in the outpatient setting as patients are less closely monitored. On the other hand, the override rate might be higher given the greater familiarity of patients to their primary care providers, whereas inpatient providers would be less likely to be familiar with a patient's past medical history and therefore may be less likely to override alerts, except in the cases of medication desensitization.

In terms of the documented override reason given that only 'definite' alerts were considered, the alerts with an override reason of 'Low risk cross sensitivity' were an interesting finding. This override reason may have resulted from alert fatigue. Since, a significant portion of the inpatient and outpatient overrides ( $73.0 \%$ of all overrides) were triggered in the context of desensitization protocols (inpatient: $68.8 \%$; outpatient: $9.1 \%$ ), it should be considered to suppress allergy alerts from these order sets to reduce the alert burden for providers.

In evaluating the appropriateness of these DAI overrides, we found a very high proportion to be appropriately overridden. These results correspond to other studies which found an appropriateness rate of DAI alerts of $96.0 \%$ and $70.9 \%$ in the inpatient and outpatient settings, respectively. ${ }^{8}$ Of greater significance than the appropriateness of the override is whether patients 
were actually harmed by CDS alerts overrides. In a study focused on DAI, 22 potential adverse drug events (ADEs) were found over a 3-month period. ${ }^{10} \mathrm{~A}$ total of 19 were confirmed to be ADEs via a physician panel discussion, of which $10(52.6 \%)$ were identified to be classified as 'significant' with 9 classified as serious ADEs. However, none of these ADEs were identified to have been preventable. In our study, no harm occurred as a result of these overrides, which is of note as there was no difference between appropriately and inappropriately overridden anaphylaxis alerts. This was because no inappropriately overridden alerts resulted in medication administration to the patient.

Similar to our study, the most common reason for overriding these DAI alerts in another publication was that the patient had previously tolerated the medication $(50.9 \%) .{ }^{9}$ However, data suggest that a patient's allergy list is updated in only $16.7 \%$ of the time, which is particularly striking if "has previously tolerated" or "patient does not have this allergy" was entered by providers..$^{10}$ The authors suggest that this may be due to reliance on patient self-report, infrequent updating of allergies, or the numerous locations where allergies may be documented in a patient's medical record.

Our study has several limitations. This study was done within one health-system and therefore the results may not be generalizable to other systems or settings. Although this was completed in a legacy, homegrown EHR, we do not expect the findings of this study to differ from what we would find in our current commercial EHR, as the same knowledge base is being used. We were not able to validate whether the documented anaphylaxis in these DAI alerts was a true reaction through lab or testing information. Our study suggests that documentation of 'anaphylaxis' is not very specific, given that a common override reason was that the patient actually did not have this allergy. In the case in which a provider overrides an alert due to the 
perception of a false-negative (i.e. override of an alert in a patient with actual anaphylaxis), there is a risk of anaphylaxis recurrence in approximately one-third of patients. ${ }^{12}$ Recommendations for improved DAI include increased accuracy of allergy documentation, with reconciliation and stewardship of a patient's documented allergic reactions, and the creation of algorithms designed to increase the specificity of allergy documentation by identifying likely causes of the underlying reaction. ${ }^{6,13}$

Inappropriate drug allergy alerts at best can waste clinicians' time, and so it is very important that the information contained in patients' drug allergy lists and the clinical evidence on drug cross-reactivity in the decision support systems is always up to date. All EHRs should collect information on allergic reactions, with anaphylaxis being a particularly important reaction. It might be helpful in the future to ask providers why they believe the reaction was anaphylaxis to enable better downstream decision-making. Additionally, removal of these alerts may be beneficial when a desensitization protocol is ordered.

\section{CONCLUSION}

We found a high rate of overrides of warnings against definite anaphylaxis, but a high proportion of these overrides were judged to be appropriate in both inpatient and outpatient settings. Desensitization protocols should be modified to bypass these warnings. Improved patient allergy reconciliation may also be beneficial in reducing the alert burden associated with these potentially critical alerts. 


\section{REFERENCES}

1. World Health Organization. International drug monitoring: the role of national centres. World Health Organ Tech Rep Ser 1972;498:1-25.

2. Johansson SG, Biever T, Dahl R, et al. Revised nomenclature for allergy for global use: Report of the Nomenclature Review Committee of the World Allergy Organization, October 2003. J Allergy Clin Immunol 2004;113:832-6.

3. Thong BY, Tan T. Epidemiology and risk factors for drug allergy. Br J Clin Pharmacol 2011;71:684-700.

4. Zhou L, Dhopeshwarkar N, Blumenthal KG et al. Drug allergies documented in electronic health records of a large healthcare system. Allergy 2016;71:1305-13.

5. Simons FE, Ebisawa M, Sanchez-Borges M, et al. 2015 update of the evidence base: World Allergy Organization anaphylaxis guidelines. World Allergy Organ J 2015;8:32.

6. Topaz M, Goss F, Blumenthal $\mathrm{K}$,et al. Towards improved drug allergy alerts: multidisciplinary expert recommendations. Int J Med Inform 2017;97:353-5.

7. Electronic Health Records (EHR) Incentive Programs. Centers for Medicare \& Medicaid Services website. Available at: https://www.cms.gov/Regulations-andGuidance/Legislation/EHRIncentivePrograms/index.html. Updated February 8, 2017. Accessed May 8, 2017.

8. Slight SP, Beeler PE, Seger DL, et al. A cross-sectional observational study of high override rates of drug allergy alerts in inpatient and outpatient settings, and opportunities for improvement. BMJ Qual Saf 2017;26:217-25.

9. Topaz M, Seger DL, Slight SP, et al. Rising drug allergy alert overrides in electronic health records: an observational retrospective study of a decade of experience. J Am Med Inform Assoc 2016;23:601-8.

10. Hsieh TC, Kuperman GJ, Jaggi T, et al. Characteristics and consequences of drug allergy allergy overrides in a computerized physician order entry system. J Am Med Inform Assoc 2004;11:482-91.

11. Kuperman GJ, Marston E, Paterno M, et al. Creating an enterprise-wide allergy repository at Partners HealthCare System. AMIA Annu Symp Proc 2003;2003:376-80.

12. Mullins RJ. Anaphylaxis: risk factors for recurrence. Clin Exp Allergy 2003;33:1033-40.

13. Cahill KN, Johns CB, Cui J, et al. Automated identification of an aspirin-exacerbated respiratory disease cohort. J Allergy Clin Immunol 2017;139:819-25.e6. 


\section{TABLES}

Table 1. Patient demographics

\begin{tabular}{|l|c|c|}
\hline & Inpatient (n=107) & $\begin{array}{c}\text { Outpatient } \\
(\mathbf{n = 1 3 )}\end{array}$ \\
\hline Age, y, median (IQR) & $63(49,72.5)$ & $66(44,81)$ \\
\hline Female, n (\%) & $84(78.5)$ & $11(84.6)$ \\
\hline Race, n (\%) & $86(80.4)$ & $10(76.9)$ \\
\hline White & $9(8.4)$ & $1(7.7)$ \\
\hline Black & $3(2.8)$ & $1(7.7)$ \\
\hline Hispanic & $1(0.9)$ & $0(0)$ \\
\hline Asian & $8(7.5)$ & $1(7.7)$ \\
\hline Other & $4.0(2.0,7.0)$ & $6.0(4.0,7.0)$ \\
\hline Number of documented allergies, n, median (IQR) & \multicolumn{2}{|l}{} \\
\hline
\end{tabular}


Table 2. Documented override reason or action with most common medications by override reason for inpatient alerts

\begin{tabular}{|c|c|}
\hline Cancel order, $n$ (\% of total) & $109(54.0)$ \\
\hline Prochlorperazine, $\mathrm{n}(\%)$ & $21(19.3)$ \\
\hline Ondansetron, $\mathrm{n}(\%)$ & $13(11.9)$ \\
\hline Acetaminophen, $\mathrm{n}(\%)$ & $8(7.3)$ \\
\hline $\begin{array}{l}\text { Administer per desensitization protocol, } n \\
(\% \text { of total) }\end{array}$ & $64(31.7)$ \\
\hline Aspirin, $\mathrm{n}(\%)$ & $13(20.3)$ \\
\hline Paclitaxel, n (\%) & $13(20.3)$ \\
\hline Carboplatin, $\mathrm{n}(\%)$ & $12(18.8)$ \\
\hline $\begin{array}{l}\text { Patient has taken previously without } \\
\text { allergic reaction/patient has tolerated } \\
\text { previously, } n(\% \text { of total) }\end{array}$ & $10(5.0)$ \\
\hline Prochlorperazine, $\mathrm{n}(\%)$ & $3(30.0)$ \\
\hline Ipratropium and albuterol, $\mathrm{n}(\%)$ & $2(20.0)$ \\
\hline Other, $\mathrm{n}(\%)$ & $5(50.0)$ \\
\hline Physician aware, n (\% of total) & $7(3.5)$ \\
\hline Ciprofloxacin, $\mathrm{n}(\%)$ & $2(28.6)$ \\
\hline Ondansetron, $\mathrm{n}(\%)$ & $2(28.6)$ \\
\hline Other, $\mathrm{n}(\%)$ & $3(42.8)$ \\
\hline Other, $n$ (\% of total) & $12(5.8)$ \\
\hline Oxycodone, n (\%) & $3(25.0)$ \\
\hline Other, $\mathrm{n}(\%)$ & $9(75.0)$ \\
\hline
\end{tabular}


Table 3. Documented override reason or action with most common medications by override reason for outpatient alerts

\begin{tabular}{|l|c|}
\hline Cancel order, n (\% of total) & $\mathbf{5 ( 3 1 . 3 )}$ \\
\hline Azithromycin, n (\%) & $3(60.0)$ \\
\hline Ibuprofen, n (\%) & $1(20.0)$ \\
\hline Nitrofurantoin, n (\%) & $1(20.0)$ \\
\hline $\begin{array}{l}\text { Patient does not have this allergy, will D/C } \\
\text { pre-existing allergy, n (\% of total) }\end{array}$ & $\mathbf{7 ( 4 3 . 8 )}$ \\
\hline Aspirin, n (\%) & $4(57.1)$ \\
\hline Butalbital-acetaminophen-caffeine, n (\%) & $1(14.3)$ \\
\hline Levofloxacin, n (\%) & $1(14.3)$ \\
\hline Tocilizumab, n (\%) & $1(14.3)$ \\
\hline $\begin{array}{l}\text { Patient has taken previously without } \\
\text { allergic reaction/patient has tolerated } \\
\text { previously, n (\% of total) }\end{array}$ & $\mathbf{3}(\mathbf{1 8 . 8 )}$ \\
\hline Morphine, n (\%) & $2(66.7)$ \\
\hline Insulin lispro, n (\%) & $1(33.3)$ \\
\hline Desensitization, n (\% of total) & $\mathbf{1 ( 6 . 1 )}$ \\
\hline Aspirin, n (\%) & $1(100)$ \\
\hline
\end{tabular}




\section{FIGURES}

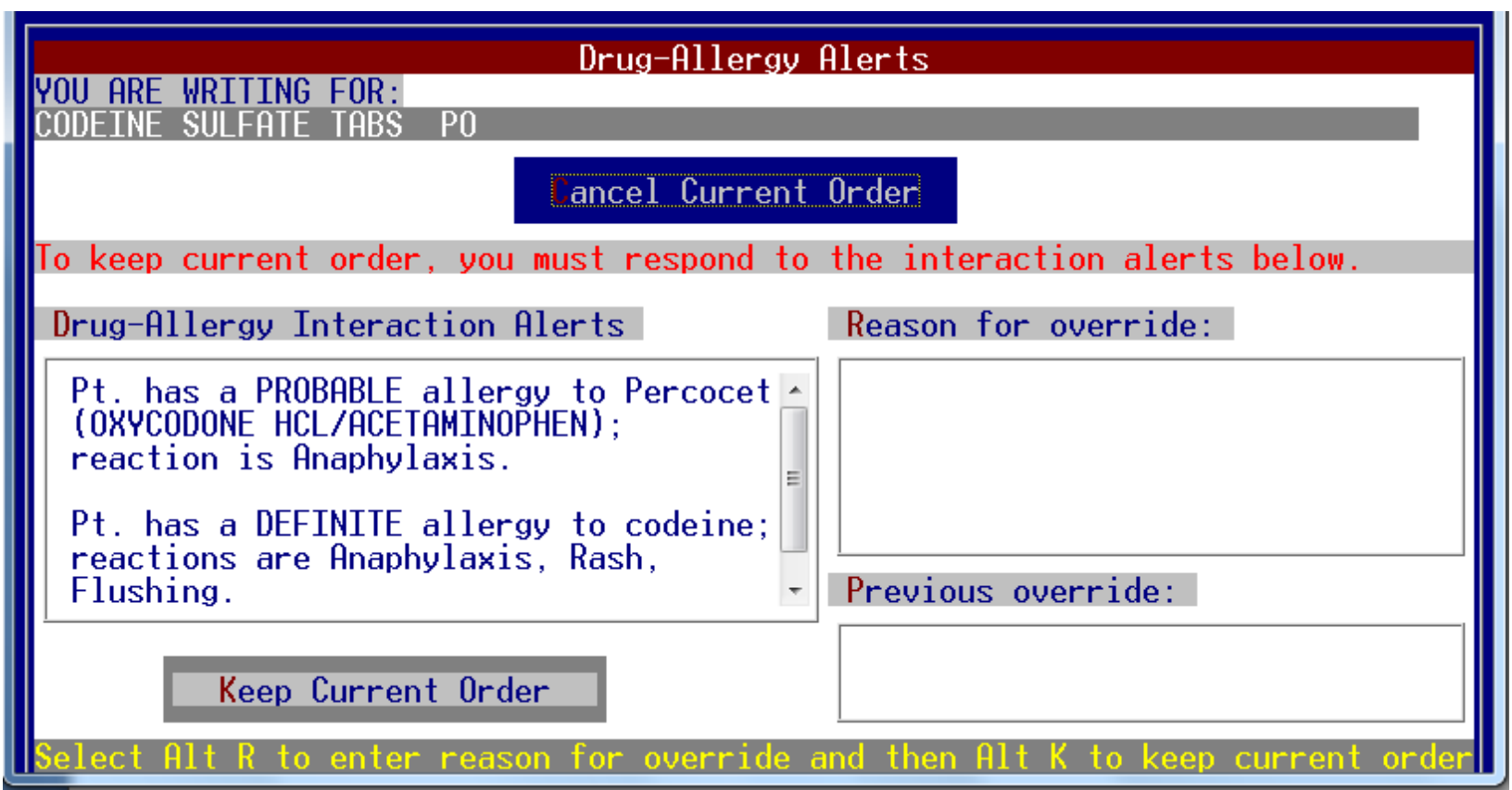

Figure 1. Drug-allergy interaction alert in the inpatient setting for a patient with anaphylaxis to codeine

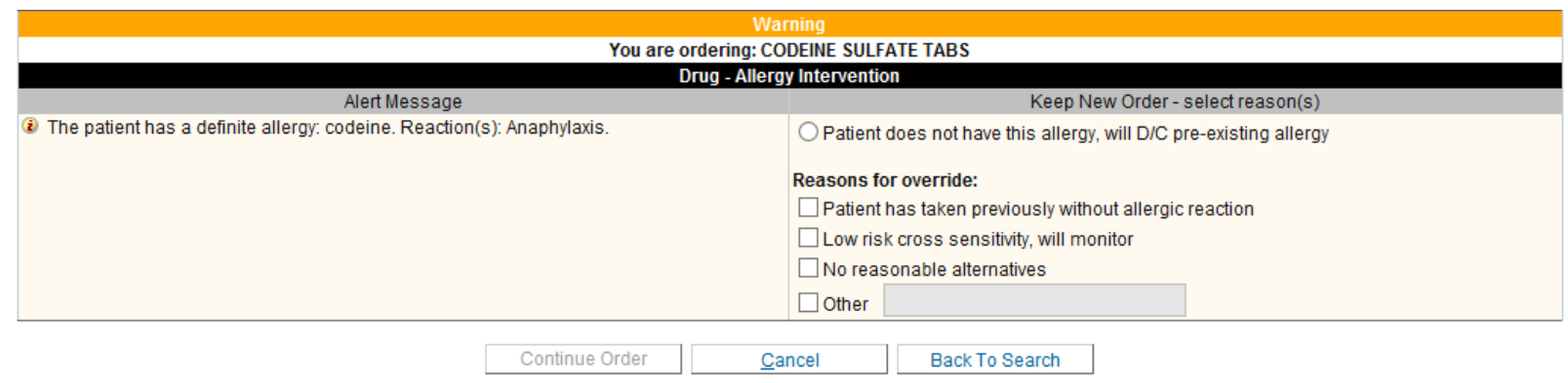

Figure 2. Drug-allergy interaction alert in the outpatient setting for a patient with anaphylaxis to codeine 